\title{
Influence of the Magnetic Field on the Graphene Conductivity
}

\author{
Sergey Viktorovich Kryuchkov, ${ }^{1,2}$, Egor Ivanovich Kukhar,1 \\ ${ }^{1}$ Volgograd State Socio-Pedagogical University, Volgograd, Russia \\ ${ }^{2}$ Volgograd State Technical University, Volgograd, Russia \\ Email: svkruchkov@yandex.ru, eikuhar@yandex.ru
}

Received June 23, 2012; revised July 30, 2012; accepted August 7, 2012

\begin{abstract}
The transversal conductivity of the gap-modification of the graphene was studied in the cases of weak nonquatizing and quantizing magnetic field. In the case of nonquantizing magnetic field the expression of the current density was derived from the Boltzmann equation. The dependence of conductivity and Hall conductivity on the magnetic field intensity was investigated. In the case of quantizing magnetic field the expression for the graphene transversal magnetoconductivity taking into account the scattering on the acoustic phonons was derived in the Born approximation. The graphene conductivity dependence on the magnetic field intensity was investigated. The graphene conductivity was shown to have the oscillations when the magnetic field intensity changes. The features of the Shubnikov-de Haas oscillations in graphene superlattice are discussed.
\end{abstract}

Keywords: Graphene; Magnetoconductivity; Magnetic Oscillations; Shubnikov-de Haaz Effect; Graphene Superlattice

\section{Introduction}

The development of the micro- and nanoelectronics requires the search of new materials and structures. Presently the investigators are attracted by the electron features of the graphene (monolayer carbon) which is obtained in the laboratory recently [1]. The heightened interest in the electronic properties of the graphene is related with the following. Firstly experimentally the electron mean free path is shown to have the order of a micrometer in graphene [1]. This fact allows the graphene using for creation of the micrometer devices working in the ballistic regime. The high electrical conductivity of the graphene makes it the prospective material for using in the nanoelectronics along with carbon nanotubes [2]. The samples of the field-effect transistors and number of other electronic devices based on it have already worked out $[3,4]$.

Secondly this material has a number of unusual properties due to it band structure peculiarities [5-7]. Nonparabolicity and non-additivity of the graphene electronic spectrum enable the appearance of a number of the nonlinear kinetic effects in this material [8-11]. Besides near the so-called Dirac points of the Brillouin zone for the gapless modification of the graphene the dispersion law is linear in the absolute value of the quasimomentum which is corresponding to the massless particles [7]. Furthermore this fact allows the graphene using for the verifying of the relativistic effects.

More recently, the electric properties of the graphene superlattice (GSL) are under investigations [12-16].

The theoretical and experimental studies of the influence of the external fields of different configuration on the graphene transport features are held recently [17-25]. The conductivity oscillations in the graphene under the spatially modulated magnetic field are investigated theoretically in [17]. The theory of the electron transport of the charge carriers with Dirac spectrum in the weak magnetic field taking into account the scattering on the charge impurity is built in [18]. In [16-22] the magnetic oscillation effects in the structures based on the graphene are studied. The magnetic field influences on the high frequency conductivity and on the electromagnetic waves absorption of the graphene are investigated in [22-25]. The quantum theory of the transverse magnetoconductivity oscillations (Shubnikov-de Haaz effect) in the twodimentional system with Dirac spectrum are studied in [21].

When the graphene is put on the substrate ( $\mathrm{SiC}$ for example) then the gap arises (so-called the gap modification of the graphene $[26,27])$. The electron spectrum of the gap modification of the graphene can be written in the view [10]:

$$
\varepsilon_{G}(\boldsymbol{p})=\sqrt{\Delta^{2}+v_{F}^{2} \boldsymbol{p}^{2}},
$$

where $\boldsymbol{p}$ is the quasimomentum, $\Delta$ is the gap semi- 
width, $v_{F}$ is the velocity on the Fermi surface.

When the graphene sheet is applied upon a periodic substrate, a superlattice (SL) is formed on the graphene surface [28]. The dispersion low of GSL was studied in [28] where the energy of electron motion near the Dirac point along the SL axis was shown to be periodically dependent on the electron quasimomentum in this direction. The electron spectrum of the graphene superlattice was written in the following model view [29]:

$$
\varepsilon_{\mathrm{GSL}}(\boldsymbol{p})=\sqrt{\Delta^{2}+v_{F}^{2} p_{y}^{2}+\frac{2 \hbar^{2} v_{F}^{2}}{d^{2}}\left(1-\cos \frac{p_{x} d}{\hbar}\right)},
$$

which is in good agree with dispersion low [28] if the following condition is performed: $\gamma=\hbar v_{F} / \Delta d \ll 1$.

In this paper the dependence of the graphene gap modification conductivity on the magnetic field intensity was investigated. Moreover the peculiarities of Shubnikov-de Haaz effect in the graphene superlattice were discussed.

Consider the graphene lying in the plane $x y$ under the crossed magnetic and electric fields so that the magnetic field intensity $\boldsymbol{H}$ is directed perpendicularly to the graphene plane and the electric field intensity $\boldsymbol{E}$ is directed along the axis $O x$.

\section{The Nonquantizing Magnetic Field Influence on the Conductivity of the Gap Modification of the Graphene}

Consider the case of the nonquantizing magnetic field: $e \hbar v_{F}^{2} H / \Delta c \leq \theta$, where $\theta$ is the electron gas temperature in energy units. Current density arising in graphene under the condition described above is calculated with the following formula:

$$
\boldsymbol{j}=-e \sum_{p} \boldsymbol{V}(\boldsymbol{p}) f(\boldsymbol{p}, t),
$$

where $\boldsymbol{V}=\partial \varepsilon_{G} / \partial \boldsymbol{p}$ is the electron velocity, $f(\boldsymbol{p}, t)$ is the nonequilibrium state function which is determined from the Boltzmann equation written in the approximation of the constant relaxation time $\tau$ :

$$
\frac{\partial f}{\partial t}-e\left(\boldsymbol{E}+\frac{1}{c}[\boldsymbol{V}, \boldsymbol{H}]\right) \cdot \frac{\partial f}{\partial \boldsymbol{p}}=-\frac{f-f_{0}}{\tau} .
$$

The solution of the kinetic Equation (4) is the following function:

$$
f(\boldsymbol{p}, t)=\frac{1}{\tau} \int_{-\infty}^{t} e^{-\frac{t-t^{\prime}}{\tau}} f_{0}\left(\boldsymbol{p}^{\prime}\left(\boldsymbol{p}, t, t^{\prime}\right)\right) \mathrm{d} t^{\prime},
$$

where $f_{0}(\boldsymbol{p})$ is the equilibrium state function. The electron momentum $\boldsymbol{p}^{\prime}\left(t^{\prime}\right)$ is the solution of the classical equation of motion:

$$
\boldsymbol{p}^{\prime}=\boldsymbol{p}-e \boldsymbol{E}\left(t^{\prime}-t\right)-\frac{e}{c}\left[\int_{t}^{t^{\prime}} \boldsymbol{V}^{\prime}\left(\boldsymbol{p}^{\prime}\right) \mathrm{d} t_{1}, \boldsymbol{H}\right] .
$$

where $\boldsymbol{p}$ is initial momentum. Solving (6) by the iterations of the small parameter $v_{F} / c \ll 1$ we obtain the following expression in linear approximation of the electric field intensity:

$$
\begin{aligned}
p_{x}= & p_{x}^{\prime}-\frac{e v_{F}^{2} H p_{y}^{\prime}\left(t-t^{\prime}\right)}{c \sqrt{\Delta^{2}+v_{F}^{2} p^{\prime 2}}} \\
& -\left(1+\frac{e H v_{F}^{4} p_{x}^{\prime} p_{y}^{\prime}\left(t-t^{\prime}\right)}{2 c\left(\Delta^{2}+v_{F}^{2} p^{\prime 2}\right)^{3 / 2}}\right) e E_{x}\left(t-t^{\prime}\right), \\
p_{y}= & p_{y}^{\prime}+\frac{e v_{F}^{2} H p_{x}^{\prime}\left(t-t^{\prime}\right)}{c \sqrt{\Delta^{2}+v_{F}^{2} p^{\prime 2}}} \\
& +\frac{e v_{F}^{2} H\left(t-t^{\prime}\right)}{2 c \sqrt{\Delta^{2}+v_{F}^{2} p^{\prime 2}}}\left(\frac{v_{F}^{2} p_{x}^{\prime 2}}{\Delta^{2}+v_{F}^{2} p^{\prime 2}}-1\right) e E_{x}\left(t-t^{\prime}\right) .
\end{aligned}
$$

Introduce the following denotations:

$$
\begin{aligned}
& a_{1}=p_{x}^{\prime}-\frac{e v_{F}^{2} H p_{y}^{\prime}\left(t-t^{\prime}\right)}{c \sqrt{\Delta^{2}+v_{F}^{2} p^{\prime 2}}}, \\
& b_{1}=-\frac{e H v_{F}^{4} p_{x}^{\prime} p_{y}^{\prime}\left(t-t^{\prime}\right)}{2 c\left(\Delta^{2}+v_{F}^{2} p^{\prime 2}\right)^{3 / 2}}, \\
& a_{2}=p_{y}^{\prime}+\frac{e v_{F}^{2} H p_{x}^{\prime}\left(t-t^{\prime}\right)}{c \sqrt{\Delta^{2}+v_{F}^{2} p^{\prime 2}}}, \\
& b_{2}=-\frac{e v_{F}^{2} H\left(t-t^{\prime}\right)\left(\Delta^{2}+v_{F}^{2} p_{y}^{\prime 2}\right)}{2 c\left(\Delta^{2}+v_{F}^{2} p^{\prime 2}\right)^{3 / 2}} .
\end{aligned}
$$

Replacing (5) - (8) to (3) and considering that $a_{1}, a_{2}$, $b_{1}$ are the odd functions of $p_{x}^{\prime}$ and $p_{y}^{\prime}$ we define the projections of current density in the linear approximation of $E_{x}$ :

$$
\begin{aligned}
j_{x}= & \frac{e^{2} v_{F}^{2} E_{x} \tau}{\Delta} \int_{0}^{\infty} x e^{-x} \mathrm{~d} x \sum_{p} f_{0}(\boldsymbol{p})\left(1+q^{2}\left(1+\frac{H^{2} x^{2}}{H_{0}^{2}\left(1+q^{2}\right)}\right)\right)^{-3 / 2} \\
& \times\left(1+q^{2}\left(1+\frac{H^{2} x^{2}}{H_{0}^{2}\left(1+q^{2}\right)}\right)+q_{x}^{2}+\frac{\left(q_{x}^{2}-q_{y}^{2}-q_{x}^{2} q_{y}^{2}-q_{y}^{4}\right) H^{2} x^{2}}{2 H_{0}^{2}\left(1+q^{2}\right)^{2}}\right),
\end{aligned}
$$




$$
\begin{aligned}
j_{y}= & \frac{e^{2} v_{F}^{2} E_{x} \tau}{\Delta} \frac{H}{H_{0}} \int_{0}^{\infty} x^{2} e^{-x} \mathrm{~d} x \sum_{p} f_{0}(\boldsymbol{p})\left(1+q^{2}\left(1+\frac{H^{2} x^{2}}{H_{0}^{2}\left(1+q^{2}\right)}\right)\right)^{-3 / 2} \\
& \times\left(\frac{H_{0}^{2}\left(1+q^{2}+2 q_{y}^{2}+2 q_{y}^{4}-2 q_{x}^{2}-2 q_{x}^{4}\right)+H^{2} x^{2} q_{y}^{2}}{2 H_{0}^{2}\left(1+q^{2}\right)^{3 / 2}}\right) .
\end{aligned}
$$

In (10) and (11) we introduced dimensionless variables: $x=\left(t-t^{\prime}\right) / \tau, \boldsymbol{q}=v_{F} \boldsymbol{p} / \Delta$ and denote $H_{0}=c \Delta / e v_{F}^{2} \tau$. Choose the equilibrium state function in the view of Boltzmann function:

$$
f_{0}(\boldsymbol{p})=A e^{-\frac{\varepsilon_{G}(\boldsymbol{p})}{\theta}}
$$

where $A$ is the constant determined from the normalization condition:

$$
\begin{aligned}
& \sum_{p} f_{0}(\boldsymbol{p})=n_{0}, \quad \text { (13) } \begin{array}{c}
\text { As a result we obtain the following expressions } \\
\text { projections of the current density: }
\end{array} \\
& j_{x}=\frac{n_{0} e^{2} v_{F}^{2} E_{x} \tau}{4 \theta(1+\theta / \Delta)} \int_{0}^{\infty} x e^{-x} \mathrm{~d} x \int_{0}^{\infty} e^{-\frac{\Delta}{\theta}(\sqrt{1+y}-1)}\left(1+y\left(1+\frac{H^{2} x^{2}}{H_{0}^{2}(1+y)}\right)\right)^{-3 / 2}\left(2+3 y+\frac{(4+3 y) y H^{2} x^{2}}{2 H_{0}^{2}(1+y)^{2}}\right) \mathrm{d} y, \\
& j_{y}=\frac{n_{0} e^{2} v_{F}^{2} E_{x} \tau}{4 \theta(1+\theta / \Delta)} \frac{H}{H_{0}} \int_{0}^{\infty} x^{2} e^{-x} \mathrm{~d} x \int_{0}^{\infty} e^{-\frac{\Delta}{\theta}(\sqrt{1+y}-1)}\left(1+y\left(1+\frac{H^{2} x^{2}}{H_{0}^{2}(1+y)}\right)\right)^{-3 / 2}\left(\frac{2 H_{0}^{2}(1+y)+H^{2} x^{2} y}{2 H_{0}^{2}(1+y)^{3 / 2}}\right) \mathrm{d} y .
\end{aligned}
$$

The components of the conductivity tensor are determined from the formulas $j_{x}=\sigma_{x x} E_{x}, j_{y}=\sigma_{x y} E_{x}$. Therefore the magnitoconductivity of the graphene is equal to:

$$
\sigma_{x x}=\sigma_{0} \int_{0}^{\infty} x e^{-x} \mathrm{~d} x \int_{0}^{\infty} e^{-\frac{\Delta}{\theta}(\sqrt{1+y}-1)}\left(1+y\left(1+\frac{H^{2} x^{2}}{H_{0}^{2}(1+y)}\right)\right)^{-3 / 2}\left(2+3 y+\frac{(4+3 y) y H^{2} x^{2}}{2 H_{0}^{2}(1+y)^{2}}\right) \mathrm{d} y
$$

Hall conductivity has the view:

$$
\sigma_{x y}=\sigma_{0} \frac{H}{H_{0}} \int_{0}^{\infty} x^{2} e^{-x} \mathrm{~d} x \int_{0}^{\infty} e^{-\frac{\Delta}{\theta}(\sqrt{1+y}-1)}\left(1+y\left(1+\frac{H^{2} x^{2}}{H_{0}^{2}(1+y)}\right)\right)^{-3 / 2}\left(\frac{2 H_{0}^{2}(1+y)+H^{2} x^{2} y}{2 H_{0}^{2}(1+y)^{3 / 2}}\right) \mathrm{d} y .
$$

where $\sigma_{0}=n_{0} e^{2} v_{F}^{2} \tau / 4 \theta(1+\theta / \Delta)$.

The conductivity tensor dependence on the magnetic field intensity is investigated numerically. The plots of the conductivity dependence on the magnetic field intensity built with the formulas (16) and (17) for the following values of parameters: $n_{0}=10^{12} \mathrm{~cm}^{-2}, \theta=0.1 \mathrm{eV}$, $\tau=10^{-12} \mathrm{~s}, v_{F}=10^{8} \mathrm{~cm} / \mathrm{s}$, are shown on the Figure 1. At low temperatures $(\theta \ll \Delta)$ and weak magnetic fields $\left(H \ll H_{0}\right)$ the formulas (16) and (17) can be written approximately in the view:

$$
\begin{aligned}
& \sigma_{x x}=\frac{n_{0} e^{2} v_{F}^{2} \tau}{\Delta}\left(1-\left(1+\frac{6 e^{2} v_{F}^{4} \tau^{2}}{c^{2} \Delta^{2}} H^{2}\right) \frac{\theta}{\Delta}\right), \\
& \sigma_{x y}=\frac{n_{0} e^{3} v_{F}^{4} \tau^{2}}{2 c \Delta^{2}} H\left(1-3\left(1+\frac{4 e^{2} v_{F}^{4} \tau^{2}}{c^{2} \Delta^{2}} H^{2}\right) \frac{\theta}{\Delta}\right) .
\end{aligned}
$$

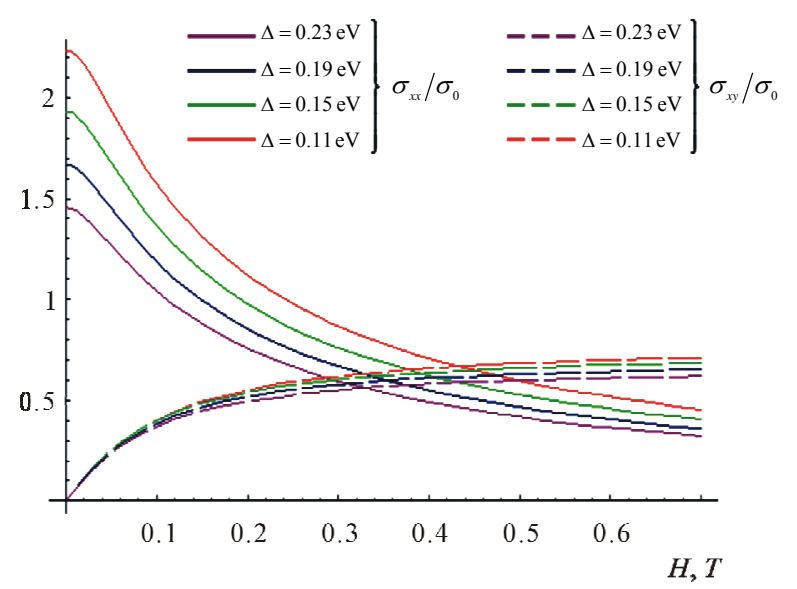

Figure 1. The conductivity tensor dependence on the magnetic field intensity at $\theta=0.1 \mathrm{eV}$ and at several values of $\Delta$. 


\section{The Oscillations of the Transversal Conductivity of the Gap Modification of the Graphene under the Quantizing Magnetic Field}

Define the graphen magnetoconductivity in the case of the quantizing magnetic field at law temperatures: $e \hbar v_{F}^{2} H / \Delta c \gg \theta$. In [21] where Shubnicov-de Haas effect was studied Landau levels were supposed to have a finite width due to the charge scattering on the lattice inhomogeneity. Herewith the particular scattering mechanism was not considered and broadening of the Landau levels was introduced as a phenomenological parameter. Calculate the transverse manetoconductivity of the gap modification of the graphene taking into account the elastic scattering of the electrons on the acoustic phonons. The wave function envelope $\psi_{G}$ of the electron is determined from the Schrödinger equation with the Hamiltonian $\hat{H}_{G}$ obtained from (1) by replacing $\boldsymbol{p} \rightarrow-i \hbar \nabla+e \boldsymbol{A} / \boldsymbol{c}$, where vector potential is equal $\boldsymbol{A}=(0, H x, 0)$. Acting twice with $\hat{H}_{G}$ to the function $\psi_{G}$ we obtain the following:

$$
-\nabla^{2} \psi_{G}-2 i \frac{x}{a_{H}^{2}} \frac{\partial \psi_{G}}{\partial y}+\frac{x^{2}}{a_{H}^{4}} \psi_{G}=\frac{\varepsilon^{2}-\Delta^{2}}{\hbar^{2} v_{F}^{2}} \psi_{G} .
$$

where $a_{H}=\sqrt{c \hbar / e H}$ is Larmor radius. The next function is the solution of the Equation (20):

$$
\psi_{G n k}(x, y) \equiv|k n\rangle=\frac{1}{\sqrt{2 L a_{H}}} e^{i k y} \Phi_{n}\left(\frac{x-x_{k}}{a_{H}}\right),
$$

where $\Phi_{n}(x)$ is the oscillator function, $k$ is the projection of the wave vector of the electron on the axis $O y$, $x_{k}=-k a_{H}^{2}, \quad n=0,1,2, \cdots, L$ is the linear size of the graphene. Eigen values of the electron energy are as fol- lows:

$$
\varepsilon_{n}=\sqrt{\Delta^{2}+\frac{\hbar^{2} v_{F}^{2}}{a_{H}^{2}}(2 n+1)} .
$$

From the cyclic conditions along the axis $\mathrm{Oy}$ : $\psi_{\mathrm{G}}(x, y)=\psi_{\mathrm{G}}(x, y+L)$ number $k$ is followed to take the values:

$$
k=\frac{2 \pi l}{L}+\frac{e}{c \hbar} A_{y}, \quad l=0,1,2, \cdots
$$

To calculate the current density in the graphene under the quantizing magnetic field we use the method developed in [30]. Consider the weak electric field $\boldsymbol{E}$ is applied along the axis $O x$. Then the components of the current density for the particles ensemble described by a density matrix $\left\langle n k|\rho| n^{\prime} k^{\prime}\right\rangle$ is determined by the formula:

$$
j_{x}=i e v_{F} \sum_{n k}(\langle k n|\rho| k n+1\rangle+\langle k n|\rho| k n-1\rangle) .
$$

The total density matrix $\hat{\boldsymbol{\rho}}_{T}$ taking into account the transition processes is determined from the equation:

$$
-i \hbar \frac{\partial \hat{\boldsymbol{\rho}}_{T}}{\partial t}=\hat{\boldsymbol{\rho}}_{T} \hat{H}_{T}-\hat{H}_{T} \hat{\boldsymbol{\rho}}_{T},
$$

where $\hat{H}_{T}$ is Hamiltonian taking into account the magnetic field, the electric field and the scattering potential $V(\boldsymbol{r})$ :

$$
\hat{H}_{T}=\hat{H}_{0}+e E_{x} x+V(\boldsymbol{r}) .
$$

The stationary density matrix $\left\langle n k|\rho| n^{\prime} k^{\prime}\right\rangle$ is the total density matrix after such a long period of time during which all transition processes disappear. In Born approximation in scattering potential $V(\boldsymbol{r})$ and in linear approximation in the electric field intensity we obtain:

$$
\langle k n|\rho| k n \pm 1\rangle=\pi i e E_{x} \sum_{n^{\prime} k^{\prime}} \frac{\partial f}{\partial \varepsilon_{n}}\left(x_{k^{\prime}}-x_{k}\right) \frac{\left\langle k n|V| k^{\prime} n^{\prime}\right\rangle\left\langle k^{\prime} n^{\prime}|V| k n \pm 1\right\rangle}{\varepsilon_{n \pm 1}-\varepsilon_{n}}\left(\delta\left(\varepsilon_{n^{\prime}}-\varepsilon_{n}\right)+\delta\left(\varepsilon_{n^{\prime}}-\varepsilon_{n \pm 1}\right)\right) .
$$

After substitution of (25) in (24) and after the some transformations we obtain the following expression for the transversal magnetoconductivity of graphene which coincides with the Titeica formula [30]:

$$
\sigma_{x x}=-\frac{\pi e^{2}}{\hbar} \sum_{n k} \sum_{n^{\prime} k^{\prime}} \frac{\partial f}{\partial \varepsilon_{n}}\left(x_{k^{\prime}}-x_{k}\right)^{2}\left|\left\langle k^{\prime} n^{\prime}|V| k n\right\rangle\right|^{2} \delta\left(\varepsilon_{n^{\prime}}-\varepsilon_{n}\right) .
$$

Consider the electrons dissipate on the acoustic phonons in graphene. Then the scattering potential can be written in the following view [31]:

$$
\begin{array}{r}
V(\boldsymbol{r})=\sum_{q} V_{q} \exp (i \boldsymbol{q} \cdot \boldsymbol{r}), \\
\sigma_{x x}=-\frac{\pi e^{2}}{\hbar} \sum_{n k} \sum_{n^{\prime} k^{\prime}} \sum_{q} V_{q}^{2} \frac{\partial f}{\partial \varepsilon_{n}}\left(x_{k^{\prime}}-x_{k}\right)^{2}\left|\left\langle k^{\prime} n^{\prime}\left|e^{i q \cdot r}\right| k n\right\rangle\right|^{2} \delta\left(\varepsilon_{n^{\prime}}-\varepsilon_{n}\right) .
\end{array}
$$

where $V_{q}=\sqrt{\hbar \Lambda^{2} q / 2 \rho v S}, \boldsymbol{q}$ is the wave vector of the acoustic phonons, $\Lambda$ is the deformation potential, $\rho$ is the surface density of graphene, $v$ is the sound velocity in graphene, $S$ is the sample area. After substitution 
From (28) the conductivity is shown to be different from zero in the case when $n=n^{\prime}$. The absolute value of the matrix element included in the formula (28) is equal:

$$
\left|\left\langle k^{\prime} n\left|e^{i q \cdot r}\right| k n\right\rangle\right|=\left|L_{n}\left(\frac{a_{H}^{2} q^{2}}{2}\right)\right| \exp \left(-\frac{a_{H}^{2} q^{2}}{4}\right) \delta_{k^{\prime} k+q_{x}}
$$

where $L_{n}(x)$ is the Laguerre polynomial. After substitution of (29) in (28) and after calculation of the sum by $k$ and $k^{\prime}$ we obtain:

$$
\begin{array}{r}
\sigma_{x x}=-\frac{e^{2} \Lambda^{2}}{4 \sqrt{2} \pi^{2} \rho v} \frac{1}{a_{H}^{3}} \sum_{n} \sum_{n^{\prime}} F_{n} \frac{\partial f}{\partial \varepsilon_{n}} \delta\left(\varepsilon_{n^{\prime}}-\varepsilon_{n}\right), \text { (30) } \begin{array}{l}
\text { mula (30). Using the Poiss } \\
\text { (30) to the following view: }
\end{array} \\
\sigma_{x x}=\frac{e^{2} \Lambda^{2}}{16 \sqrt{2} \pi^{2} \rho v \theta \hbar^{2} v_{F}^{2}} \frac{1}{a_{H}} \sum_{k=-\infty}^{\kappa=+\infty} \int_{0}^{\infty} F_{n} \varepsilon(n) \operatorname{ch}^{-2} \frac{\varepsilon(n)-\mu}{2 \theta} e^{2 \pi i k n} \mathrm{~d} n,
\end{array}
$$

where $\mu$ is the chemical potential, $\theta$ is the electron gas temperature. The factor $F_{n}$ represents a slowly varying function of $n$ in compared with the oscillatory where

$$
F_{n}=\int_{0}^{\infty} x^{4} L_{n}^{2}\left(x^{2}\right) \exp \left(-x^{2}\right) \mathrm{d} x
$$

If the conductivity oscillations are small in compared with the non-oscillatory part then it can be taken into account in one of the sum (30) only. At low temperatures electron gas is degenerate. Hence Fermi-Dirac state function is needed to use as the function $f(\varepsilon)$ in the formula (30). Using the Poisson formula [32] we transform

$$
\sigma_{x x}=\frac{\sigma_{1}}{2} \sqrt{\frac{H_{1}}{H}} \sum_{\kappa=-\infty}^{\kappa=+\infty} \exp \left(\pi i \kappa\left(\frac{\mu^{2}}{\Delta^{2}}-1\right) \frac{H_{1}}{H}-\pi i \kappa\right)_{-\infty}^{+\infty} \exp \left(\frac{\pi i \kappa \mu \theta}{\Delta^{2}} \frac{H_{1}}{H} y\right) c h^{-2} y \mathrm{~d} y,
$$

where $H_{1}=c \Delta^{2} / e \hbar v_{F}^{2}, \sigma_{1}=e^{2} \Lambda^{2} \mu^{2} / 4 \sqrt{2} \pi^{2} \rho v \Delta \hbar^{3} v_{F}^{3}$. Since $\int_{-\infty}^{+\infty} e^{i a y} \operatorname{ch}^{-2} y \mathrm{~d} y=\pi a \operatorname{sh}^{-1}\left(\frac{\pi a}{2}\right)$, so the formula (33) can be rewritten as:

$$
\sigma_{x x}=\sigma_{1} \sqrt{\frac{H_{1}}{H}}\left(1+\frac{\pi^{2} \mu \theta}{\Delta^{2}} \frac{H_{1}}{H} \sum_{\kappa=1}^{\infty}(-1)^{\kappa} \kappa \operatorname{sh}^{-1}\left(\frac{\pi^{2} \kappa \mu \theta}{2 \Delta^{2}} \frac{H_{1}}{H}\right) \cos \left(\pi \kappa\left(\frac{\mu^{2}}{\Delta^{2}}-1\right) \frac{H_{1}}{H}\right)\right),
$$

On the Figure 2 the graphene conductivity dependences on the magnetic field intensity constructed by the formula (34) are shown.

\section{The Shubnikov-de Haaz Effect in the Superlattice Based on the Graphene}

To calculate conductivity of GSL we have to define the energy of electron in the GSL under the quantizing magnetic field. The wave function envelope $\psi_{\mathrm{GSL}}$ of the electron is determined from the Schrödinger equation with the Hamiltonian $\hat{H}_{\text {GSL }}$ obtained from (2) by replacing $\boldsymbol{p} \rightarrow-i \hbar \nabla+e \boldsymbol{A} / \boldsymbol{c}$, where vector potential is chosen in form $\boldsymbol{A}=(-H y, 0,0)$. Acting twice with $\hat{H}_{\mathrm{GSL}}$ to the wave function $\psi_{\mathrm{GSL}}$ we obtain the following equation:

$$
\begin{aligned}
& {\left[\Delta^{2}-\hbar^{2} v_{F}^{2} \frac{\partial^{2}}{\partial y^{2}}+\frac{2 \hbar^{2} v_{F}^{2}}{d^{2}}\left(1-\cos \left(\frac{d y}{a_{H}^{2}}+i d \frac{\partial}{\partial x}\right)\right)\right] \psi_{\mathrm{GSL}}} \\
& =\varepsilon^{2} \psi_{\mathrm{GSL}} .
\end{aligned}
$$

The solution of (35) is found in the view [33]:

$$
\psi_{\mathrm{GSL}}(x, y)=e^{i k x} F(y) .
$$

Replacing (36) in (35) we have Mathieu equation:

$$
-\frac{\partial^{2} F}{\partial \xi^{2}}-2 q F \cos 2 \xi=\Gamma(q) F
$$

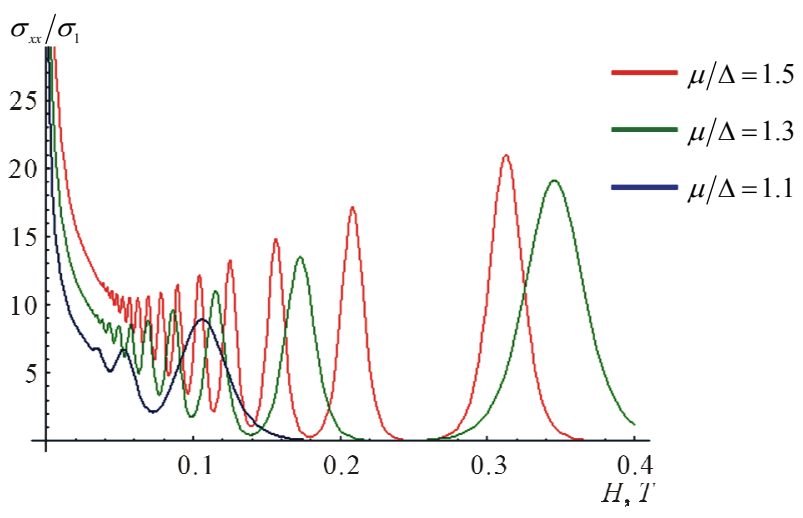

Figure 2. Graphene transversal conductivity dependence on the magnetic field intensity at $\mu / \Delta=1.1, \theta / \Delta=0.01$ and at several values of $\boldsymbol{\mu} / \boldsymbol{\theta}$. 
where $\xi=\frac{d}{2 a_{H}^{2}} y-\frac{k d}{2}, q=\frac{4 a_{H}^{4}}{d^{4}}$,

$\Gamma(q)=\frac{q d^{2}}{\hbar^{2} v_{F}^{2}}\left(\varepsilon^{2}-\Delta^{2}-\frac{2 \hbar^{2} v_{F}^{2}}{d^{2}}\right)$. If $q \gg 1$ then follow- ing expression for the energy is obtained [33]:

$$
\varepsilon_{n \kappa}=\Delta \sqrt{\alpha_{n}(q)+\beta_{n}(q)(1-\cos \kappa)},
$$

where $-\pi<\kappa<\pi, n=0,1,2, \cdots$, functions $\alpha_{n}(q)$ and $\beta_{n}(q)$ have the view:

$$
\begin{aligned}
\alpha_{n}(q)= & 1+2 \gamma^{2}+\frac{2 \gamma^{2}}{\sqrt{q}}\left[\sqrt{q} \exp \left(-\frac{1}{2 \sqrt{q}}\right) L_{n}\left(\frac{1}{\sqrt{q}}\right)+n+\frac{1}{2}\right], \\
\beta_{n}(q)= & \frac{4 \gamma^{2}}{\sqrt{q}} \exp \left(-\frac{\pi^{2} \sqrt{q}}{2}\right)\left[\left(\sqrt{q} \exp \left(-\frac{1}{2 \sqrt{q}}\right) L_{n}\left(\frac{1}{\sqrt{q}}\right)-\frac{1}{2}-n\right) L_{n}\left(\pi^{2} \sqrt{q}\right)\right. \\
& \left.+\sqrt{q} \exp \left(-\frac{1}{2 \sqrt{q}}\right) L_{n}\left(\pi^{2} \sqrt{q}+\frac{1}{\sqrt{q}}\right)+\frac{-1+\pi^{2} \sqrt{q}}{2} L_{n}\left(\pi^{2} \sqrt{q}\right)+L_{n}^{1}\left(\pi^{2} \sqrt{q}\right)\right],
\end{aligned}
$$

where $L_{n}^{m}(x)$ is adjoint Laguerre polynomial.

The magnetoconductivity of GSL can be estimated using formula (30) and making there the following changes:

$$
\begin{array}{ll}
\varepsilon_{n} \rightarrow \varepsilon_{n \kappa}, \sum_{n} \rightarrow \sum_{n \kappa} & \begin{array}{l}
\text { where } \sigma_{2} \sim n_{0} e^{2} \Lambda^{2} / \rho v \Delta^{2}, f(\varepsilon) \text { is } \\
\text { function. After some transformations }
\end{array} \\
\sigma_{x x} \sim \frac{\sigma_{2}}{\theta q^{3 / 4}} \sum_{l} \int_{0}^{\infty} e^{i \pi n l} \mathrm{~d} n \int_{0}^{\infty} \mathrm{ch}^{-2}\left(\frac{\varepsilon-\mu}{2 \theta}\right) \frac{\varepsilon^{2} \mathrm{~d} \varepsilon}{\left(\varepsilon^{2}-\Delta^{2} \alpha_{n}(q)\right)\left(\Delta^{2}\left(2 \beta_{n}(q)+\alpha_{n}(q)\right)-\varepsilon^{2}\right)} .
\end{array}
$$

If temperature is equal to zero then:

$$
\sigma_{x x} \sim \sigma_{2} \frac{4 \mu^{2}}{q^{3 / 4}} \sum_{l} \int_{0}^{\infty} \frac{e^{i \pi n l} \mathrm{~d} n}{\left(\mu^{2}-\Delta^{2} \alpha_{n}(q)\right)\left(\Delta^{2}\left(2 \beta_{n}(q)+\alpha_{n}(q)\right)-\mu^{2}\right)} .
$$

\section{Conclusions}

In the case of weak magnetic fields when the quantizing is not manifested the graphene magnetoconductivity $\sigma_{x x}$ is seen from the Figure 1 and from the formula (18) to decrease when the magnetic field intensity increases. It is related with the Larmour radius decreases and as a consequence with the electron localization increases. The formulas (14) and (15) should be noted to cease to be valid for intense magnetic fields because it was derived from the motion Equation (6) which is solved by the iterations with the parameter $v_{F} / c$ in turn. Such method is justified for small $H$.

In strong quantizing magnetic fields for graphene as well as for degenerate bulk semiconductors there are oscillations of the transverse magnetoconductivity due to the nonmonotonic dependence of the density of states on the energy and are periodic in the inverse magnetic field. However the oscillation period is not proportional to $\mu$ than that of materials with a quadratic dispersion law and has a more complicated dependence on $\mu$. For the gap modification of graphene in the case when $\mu \gg \theta$ the oscillation period is seen from the formulas (34) to be proportional to the difference $\mu^{2}-\Delta^{2}$. Thus the values $\Delta$ changing enable to control the magnetoconductivity oscillation period. The same result was obtained in [21]. However, it should be mentioned the expression (34) for the conductivity does not include the phenomenological Landau level width [21].

In GSL the dependence of the magnetic oscillations on $\mu$ is seen from the formula (43) to be more difficult than that of graphene and bulk semiconductor. Obtaining an explicit view of such dependence is the subject of further research.

For strong magnetic field $H$ the nonoscillatory part is seen from the formula (34) to decrease when the magnetic field intensity decreases as $1 / \sqrt{H}$ unlike the materials with a quadratic dispersion law where $\sigma_{x x} \sim 1 / H^{2}$ in strong magnetic fields [30]. The same result $\left(\sigma_{x x} \sim 1 / \sqrt{H}\right)$ is obtained at absolute zero of temperature whereas formula (16) gives the dependence $1 / H$ the intense magnetic fields. Such difference is ex- 
plained by the formula (16) ceases to be true when intensity $H$ increases.

\section{Acknowledgements}

The work was supported by the RFBR grant No. 10-0297001-p povolgie a and was performed within the program "The development of science potential of the High Education".

\section{REFERENCES}

[1] K. S. Novoselov, A. K. Geim, S. V. Morozov, D. Jiang, Y. Zhang, S. V. Dubonos, I. V. Grigorieva and A. A. Firsov, "Electric Field Effect in Atomically Thin Carbon Films," Science, Vol. 306, No. 5696, 2004, pp. 666-669. doi:10.1126/science. 1102896

[2] J. Milton Pereira Jr., P. Vasilopoulos and F. M. Peeters, "Graphene-Based Resonant-Tunneling Structures," Applied Physics Letters, Vol. 90, No. 13, 2007, pp. 132122132125. doi:10.1063/1.2717092

[3] Z. Chen, Y.-M. Lin, M. J. Rooks and P. Avouris, "Graphene Nano-Ribbon Electronics," Physica E, Vol. 40, No. 2, 2007, pp. 228-234. doi:10.1016/j.physe.2007.06.020

[4] Y. Q. Wu, P. D. Ye, M. A. Capano, Y. Xuan, Y. Sui, M. Qi, J. A. Cooper, T. Shen, D. Pandey, G. Prakash and R. Reifenberger, "Top-Gated Graphene Field-Effect-Transistors Formed by Decomposition of SiC," Applied Physics Letters, Vol. 92, No. 9, 2008, Article ID: 092102. doi:10.1063/1.2889959

[5] A. H. C. Neto, F. Guinea, N. M. R. Peres, K. S. Novoselov and A. K. Geim, "The Electronic Properties of Graphene," Reviews of Modern Physics, Vol. 81, No. 1, 2009, pp. 109-162. doi:10.1103/RevModPhys.81.109

[6] S. Reich, J. Maultzsch, C. Thomsen and P. Ordejon, "Tight-Binding Description of Graphene," Physical Review B, Vol. 66, No. 3, 2002, pp. 035412-035417.

[7] P. R. Wallace, "The Band Theory of Graphite," Physical Review, Vol. 71, No. 9, 1947, pp. 622-634. doi:10.1103/PhysRev.71.622

[8] S. A. Mikhailov, "Nonlinear Cyclotron Resonance of a Massless Quasiparticle in Graphene," Physical Review B, Vol. 79, No. 24, 2009, pp. 241309-241313. doi:10.1103/PhysRevB.79.241309

[9] D. V. Zav'yalov, S. V. Kryuchkov and E. V. Marchuk, "On the Possibility of Transverse Current Rectification in Graphene," Technical Physics Letters, Vol. 34, No. 11, 2008, pp. 915-917. doi:10.1134/S1063785008110047

[10] D. V. Zav'yalov, V. I. Konchenkov and S. V. Kryuchkov, "Mutual Rectification of Alternating Currents Induced by Electromagnetic Waves in Graphene," Physics of the Solid State, Vol. 51, No. 10, 2009, pp. 2157-2160. doi:10.1134/S1063783409100278

[11] D. V. Zav'yalov, V. I. Konchenkov and S. V. Kryuchkov, "Influence of a Magnetic Field on the Mutual Rectification of Alternating Currents Induced by Electromagnetic Waves in Graphene," Physics of the Solid State, Vol. 52, No. 4, 2010, pp. 800-804. doi:10.1134/S1063783410040219
[12] L. A. Chernozatonskii, P. B. Sorokin, E. E. Belova, I. Bryuning and A. S. Fedorov, "Metal-Semiconductor (Semimetal) Superlattices on a Graphite Sheet with Vacancies," JETP Letters, Vol. 84, No. 3, 2006, pp. 115-118. doi: $10.1134 / \mathrm{S} 0021364006150033$

[13] L. A. Chernozatonskii, P. B. Sorokin, E. E. Belova, I. Bryuning and A. S. Fedorov, "Superlattices Consisting of 'Lines' of Adsorbed Hydrogen Atom Pairs on Graphene," JETP Letters, Vol. 85, No. 1, 2007, pp. 77-81. doi:10.1134/S002136400701016X

[14] F. Guinea, M. I. Katsnelson and M. A. H. Vozmediano, "Midgap States and Charge Inhomogeneities in Corrugated Graphene," Physical Review, Vol. 77, No. 7, 2008, pp. 075422-075429. doi:10.1103/PhysRevB.77.075422

[15] H. Sevincli, M. Topsakal and S. Ciraci, "Superlattice Structures of Graphene-Based Armchair Nanoribbons," Physical Review, Vol. 78, No. 24, 2008, pp. 245402-245409. doi:10.1103/PhysRevB.78.245402

[16] M. R. Masir, P. Vasilopulos and F. M. Peeters, “Tunneling, Conductance, and Wavevector Filtering through Magnetic Barriers in Bilayer Graphene," Physical Review, Vol. 79, No. 3, 2009, Article ID: 035409. doi:10.1103/PhysRevB.79.035409

[17] M. Tahir and K. Sabeeh, "Quantum Transport of Dirac Electrons in Graphene in the Presence of a Spatially Modulated Magnetic Field," Physical Review B, Vol. 77, No. 19, 2008, pp. 195421-195426. doi:10.1103/PhysRevB.77.195421

[18] X.-Z. Yan and C. S. Ting, "Magnetoconductivity of Dirac Fermions in Graphene under Charged Impurity Scatterings," New Journal of Physics, Vol. 11, No. 9, 2009, Article ID: 093026. doi:10.1088/1367-2630/11/9/093026

[19] T. Shen, Y. Q. Wu, M. A. Capano, L. P. Rokhinson, L. W. Engel and P. D. Ye, "Magnetoconductance Oscillations in Graphene Antidot Arrays," Applied Physics Letters, Vol. 93, No. 12, 2008, pp. 122102-122110. doi: $10.1063 / 1.2988725$

[20] J. Jobst, D. Waldmann, F. Speck, R. Hirner, D. K. Maude, T. Seyller and H. B. Weber, "Quantum Oscillations and Quantum Hall Effect in Epitaxial Graphene," Physical Review B, Vol. 81, 2010, pp. 195434-195440. doi:10.1103/PhysRevB.81.195434

[21] S. G. Sharapov and V. P. Gusynin, "Magnetic Oscillations in Planar Systems with the Dirac-Like Spectrum of Quasiparticle Excitations II: Transport Properties," Physical Review B, Vol. 71, No. 12, 2005, pp. 125124-125132.

[22] K. Y. Bliokh, "Weak Antilocalization of Ultrarelativistic Fermions," Physics Letters A, Vol. 344, No. 2-4, 2005, pp. 127-130. doi:10.1016/j.physleta.2005.06.062

[23] V. P. Gusynin, S. G. Sharapov and J. P. Carbotte, "Dirac Quasiparticles in the Magneto-Optical Response of Graphene," Journal of Physics: Condensed Matter, Vol. 19, 2007, Article ID: 026222. doi:10.1088/0953-8984/19/2/026222

[24] N. M. R. Peres, F. Guinea and A. H. C. Neto, "Electronic Properties of Disordered Two-Dimensional Carbon," Physical Review B, Vol. 73, 2006, pp. 125411-125434.

[25] V. P. Gusynin and S. G. Sharapov, "Transport of Dirac 
Quasiparticles in Graphene: Hall and Optical Conductivities," Physical Review B, Vol. 73, No. 24, 2006, pp. 245411245429. doi:10.1103/PhysRevB.73.245411

[26] S. Y. Zhou, G.-H. Gweon, A. V. Fedorov, P. N. First, W. A. de Heer, D.-H. Lee, F. Guinea, A. H. C. Neto and A. Lanzara, "Substrate-Induced Band Gap Opening in Epitaxial Graphene," Nature Materials, Vol. 6, No. 10, 2007, pp. 770-775. doi:10.1038/nmat2003

[27] A. Mattausch and O. Pankratov, "Ab Initio Study of Graphene on SiC," Physical Review Letters, Vol. 99, No. 7, 2007, Article ID: 076802. doi:10.1103/PhysRevLett.99.076802

[28] P. V. Ratnikov, "Superlattice Based on Graphene on a Strip Substrate," JETP Letters, Vol. 90, No. 6, 2009, pp. 469-474. doi:10.1134/S0021364009180143

[29] S. V. Kryuchkov, E. I. Kuhar and V. A. Yakovenko, "Effect of the Mutual Rectification of Two Electromagnetic Waves with Perpendicular Polarization Planes in a Su- perlattice Based on Graphene," Bulletin of the Russian Academy of Sciences: Physics, Vol. 74, No. 12, 2010, pp. 1679-1681. doi:10.3103/S1062873810120129

[30] E. Adams and T. Holstein, "Quantum Theory of Transversal Galvanic-Magnetic Phenomena," Journal of Physics and Chemistry of Solids, Vol. 10, No. 4, 1959, pp. 254-297. doi:10.1016/0022-3697(59)90002-2

[31] E. H. Hwang and S. D. Sarma, "Dielectric Function, Screening, and Plasmons in Two-Dimensional Graphene," Physical Review B, Vol. 75, No. 20, 2007, pp. 205418-205424.

[32] L. D. Landau and E. M. Lifshits, "Physical Kinetics," Physical and Mathematical Literature, Moscow, 2001, pp. 462-466.

[33] A. G. Zhylich, "The Energy Spectrum of Electrons and Optical Properties of the Superlattice in a Magnetic Field," Physics of the Solid State, Vol. 36, No. 3, 1994, pp. 792 804. 